\title{
Erratum to: An Incompressible 2D Didactic Model with Singularity and Explicit Solutions of the 2D Boussinesq Equations
}

Dongho Chae, Peter Constantin, and Jiahong Wu

\section{Erratum to: J. Math. Fluid Mech. \\ DOI 10.1007/s00021-014-0166-5}

The sentence on line five, page four of our paper, namely, "It is not difficult to see that (2.1) is locally well posed if $\psi_{0} \in H^{s}\left(\mathbb{R}^{2}\right)$ with $s>3$ and $\theta_{0}=-\partial_{x_{2}} \psi_{0} \in L^{1}\left(\mathbb{R}^{2}\right)$ ", is incorrect. In fact, the sentence refers to a result that would be difficult to prove, and we actually doubt it is true. The well-posedness is very likely to hold in more complicated spaces, but the proof would take more space than the artificial nature of the example would warrant. The likely proof would be entirely similar to the recent proofs [1] of well posedness of Prandtl and hydrostatic equations, which require either a one-sided sign condition on the initial data or analyticity in one space variable and Sobolev regularity in the other.

\section{Reference}

[1] Kukavica, I., Masmoudi, N., Vicol, V., Wong, T.-K.: On the local well-posedness of the Prandtl and the hydrostatic Euler equations with multiple monotonicity regions. arXiv:1402.1984[math.AP]

\section{Dongho Chae}

Department of Mathematics

College of Natural Science

Chung-Ang University

Seoul 156-756, South Korea

e-mail:dchae@cau.ac.kr

and

Peter Constantin

Program in Applied and Computational Mathematics

Princeton University

Fine Hall, Washington Road

Princeton, NJ 08544-1000, USA.

e-mail: const@math.princeton.edu

(published online: April 1, 2014)

\section{Jiahong $\mathrm{Wu}$}

Department of Mathematics

Oklahoma State University

401 Mathematical Sciences

Stillwater, OK 74078, USA

and

Department of Mathematics,

Chung-Ang University

Seoul 156-756, Republic of Korea

e-mail: jiahong@math.okstate.edu

The online version of the original article can be found under doi:10.1007/s00021-014-0166-5. 